\title{
Black hole entanglement entropy regularized in a freely falling frame
}

\author{
Ted Jacobson*11 and Renaud Parentani垖 \\ *Department of Physics \\ University of Maryland \\ College Park, MD 20742-4111, USA \\ †Laboratoire de Physique Théorique \\ CNRS UMR 8627, Université Paris-Sud \\ 91405 Orsay Cedex, France
}

\begin{abstract}
We compute the black hole horizon entanglement entropy $S_{E}$ for a massless scalar field, first with a hard cutoff and then with high frequency dispersion, both imposed in a frame that falls freely across the horizon. Using WKB methods, we find that $S_{E}$ is finite for a hard cutoff or super-luminal dispersion, because the mode oscillations do not diverge at the horizon and the contribution of high transverse momenta is cut off by the angular momentum barrier. For sub-luminal dispersion the entropy depends on the behavior at arbitrarily high transverse momenta. In all cases it scales with the horizon area. For the hard cutoff it is linear in the cutoff, rather than quadratic. This discrepancy from the familiar result arises from the difference between the free-fall frame and the static frame in which a cutoff is usually imposed. In the superluminal case the entropy scales with a fractional power of the cutoff that depends on the index of the dispersion relation. Implications for the possible relation between regularized entanglement entropy and the Bekenstein-Hawking entropy are discussed. An appendix provides an explicit derivation of the entangled, thermal nature of the near-horizon free fall vacuum for a dispersive scalar field in four dimensions.
\end{abstract}

\footnotetext{
${ }^{1}$ E-mail: jacobson@umd.edu

${ }^{2}$ E-mail: Renaud.Parentani@th.u-psud.fr
} 


\section{Introduction}

All indications suggest that the Bekenstein-Hawking entropy of a black hole

$$
S_{\mathrm{BH}}=\frac{A}{4 L_{p}^{2}}
$$

is true thermodynamic entropy [1, 2, 3]. (Here $A$ is the area of the horizon and $L_{p}$ the Planck length.) At the microscopic level it must therefore correspond to the logarithm of the number of thermodynamically relevant states. One can write down a formal path integral expression for the partition function and entropy of the quantum gravitational statistical ensemble [4], which in the classical approximation yields precisely this entropy, however the microscopic nature of the states thereby counted remains obscure.

It would appear that an unavoidable contribution to the entropy budget is the entanglement entropy $S_{E}$ across the horizon of quantum fields propagating on the black hole background [5, 6]. The entanglement entropy arises from the spacelike correlations in the freely falling vacuum (giving rise to Hawking radiation), on account of which the restriction to the region outside the horizon is a mixed state. This can also be thought of as entropy of the thermal atmosphere of the black hole [7, 8], particularly referring to the near-horizon region where most of the entropy is localized. It is difficult to see any rationale for omitting the entanglement/atmosphere entropy. However, in the standard semiclassical evaluation it diverges due to the infinite density of states at the horizon.

One can only conclude that the divergence in $S_{E}$ must be somehow cut off by quantum gravity effects. A simple dimensional analysis of the geometry suggests that when cut off at Planck scale, the entanglement entropy across the horizon will scale as $A / L_{p}^{2}$, just like the Bekenstein-Hawking entropy. If so, then $S_{E}$ would make an important contribution to $S_{B H}$. This presents a "species problem," since $S_{E}$ depends on the number and nature of quantum fields, whereas the Bekenstein-Hawking entropy is universally given by $A / 4 L_{p}^{2}$.

A natural solution to this problem may exist however [9, 10]: the value of the Planck length $L_{p}=\left(\hbar G / c^{3}\right)^{1 / 2}$ appearing in $S_{B H}$ involves the low energy gravitational constant $G$, whose value should depend, though renormalization effects, on the quantum matter fields. The shift of $1 / G$ produced by the matter fields could induce a shift of $S_{B H}$ that corresponds precisely to $S_{E}$. The formal arguments of Refs. [9, 10] support this possibility, as do explicit regularized computations for free field theory such as in [11]. However, mismatches seem to arise for contributions of vector fields [12] and 
in odd dimensional spacetimes [13. On the other hand, very general arguments [14, 15] indicate that if one focuses not on entanglement entropy but only on the contribution of matter to the entropy derived from the thermal partition function for gravity plus matter (which may be the same thing in some cases), then the relation between black hole entropy and renormalization of $G$ is universal. A purely thermodynamic argument [16] suggests the even stronger statement that $G$ is wholly determined by the entropy of local Rindler horizons.

Previous studies of black hole entanglement entropy with a physically motivated regulator (as opposed to a technical regulator such as PauliVillars) have adopted a cutoff scheme where a highest momentum [6] or shortest distance [7] was imposed in the static frame. In both cases it was found that the entropy scales as $A / \epsilon^{2}$ where $\epsilon$ is the cutoff length. However, the equivalence principle suggests that if there is a cutoff on the freely falling vacuum imposed by quantum gravity it should be physically determined in a freely falling frame.

The aim of this paper is to investigate the nature of the regulated entanglement entropy when a freely falling momentum cutoff $\Lambda$ is imposed. We shall consider both a hard momentum cutoff, and a cutoff imposed by the introduction of dispersion at high wavevectors, and we carry out the analysis using WKB methods. We find that unlike what is found in the static frame, the entropy scales linearly with the cutoff as $A \kappa_{v} \Lambda$ for a hard cutoff, and with a similar but more complicated scaling law in the case of dispersion. In this expression $\kappa_{v}$ is the surface gravity measured in the freely falling frame in which $\Lambda$ has been defined. When that frame is at rest at infinity, $\kappa_{v}$ reduces to the standard surface gravity.

A surprising conclusion may be drawn from our results. If the freely falling cutoff $\Lambda$ is identified with the Planck mass, then the regulated entanglement entropy is negligibly small compared to the Bekenstein-Hawking entropy for macroscopic black holes. This would provide a different resolution to the species problem mentioned above (unless the number of species were of order $M_{p} / \kappa_{v}$ ). On the other hand, as argued in [17, 18, it is also possible that the cutoff arises from quantum gravitational effects in the black hole background, and that its value equals the Planck mass when measured in the static frame 3 Such a cutoff would appear as $\Lambda \sim M_{p}^{2} / \kappa_{v}$ in the freefall frame, and the regulated entropy would be of order $A / L_{p}^{2}$ after all. We

\footnotetext{
${ }^{3}$ If quantum horizon fluctuations fuzz out the horizon over the much longer scale $\left(R_{s} L_{p}^{2}\right)^{1 / 3}$, as suggested in [19, 20, 21, then the question arises whether the entropy is cut off at this scale or instead if the cutoff "floats" with the fluctuations.
} 
shall return to a discussion of this issue at the end of the paper.

Whatever the regularization scheme one chooses, the divergence in the entanglement entropy can be traced to two distinct sources. First, the density of modes at fixed frequency and angular momentum diverges due to an infinite number of oscillations as the horizon is approached. Second, there is the sum over all angular momenta. In our approach, we find that in the case of both the hard cutoff and dispersion the WKB mode density is regulated. In effect, the dispersion introduces a maximum radial wave number contributing to the thermal atmosphere of the black hole. We further find that the mode density vanishes at a finite angular momentum in the case of a hard cutoff or super-luminal dispersion, while it decreases to zero asymptotically at large angular momenta in the sub-luminal case. The angular momentum sum is therefore finite in the former cases, while in the latter case it depends on the dispersion at asymptotically large momenta and cannot be determined within the WKB approximation because of the asymptotically vanishing mode density.

The effect of sub-luminal dispersion on entanglement entropy has been previously investigated in Ref. [22]. There the dispersion was introduced in the static (accelerated) frame of the black hole, rather than in the freely falling frame. In that case, there is a piling up of transplanckian modes at some radius greater than the horizon radius, so the mode density at fixed angular momentum remains infinite and therefore the entropy diverges. This is not the result reported in [22, where it was instead concluded that the entropy is finite. We believe this conclusion was based on an incorrect evaluation of the entropy.

In following sections of this paper we first review the nature of the divergent entanglement entropy in the usual relativistic case. Next we introduce a hard momentum cut-off in the freely falling frame and analyze how it regulates the divergences. Finally we introduce dispersion at high momenta also in the freely falling frame, and analyze its impact on the divergences of the entanglement entropy. A long appendix provides details on the field modes, the entangled, thermal nature of the free fall vacuum, and the WKB evaluation of the mode density governing the entanglement entropy.

\section{Thermal atmosphere of a black hole}

We consider nonrotating, static black hole spacetimes and adopt PainlevéGullstrand (PG) coordinates since they are adapted to the free-fall frame in which the momentum cutoff or dispersion will be introduced. A free-fall 
frame is defined by a family of radial infalling geodesics all with the same velocity at infinity. The corresponding PG line element in four dimensions takes the form

$$
d s^{2}=d \tau^{2}-(d \rho-v(\rho) d \tau)^{2}-r(\rho)^{2}\left(d \theta^{2}+\sin ^{2} \theta d \phi^{2}\right),
$$

in units with the speed of light $c=1$ (see e.g. Ref. [23]). The spacetime has $\tau$-translation and rotational symmetry. The coordinate $\tau$ differs from the Schwarzschild-like time by a constant scaling and an additive function of $\rho$. The function $v(\rho)$ is the velocity $d \rho / d \tau$ of the radial geodesics, along which $\tau$ measures the proper time. These geodesics are orthogonal to the surfaces of constant $\tau$, on which $\rho$ measures radial proper distance. We consider the case $v(\rho)<0$, so that these geodesics are infalling. A surface $\rho=\rho_{H}$ on which $v^{2}\left(\rho_{H}\right)=1$ is lightlike and is a future event horizon, i.e. no timelike curve can exit the interior region $\rho<\rho_{H}$.

Expanding around the horizon one has

$$
v(\rho)=-1+\kappa_{v}\left(\rho-\rho_{H}\right)+\cdots .
$$

As we shall see, the non-linear terms are not relevant to the entropy. The constant $\kappa_{v}$ depends on the free-fall frame at the horizon.4 It is equal to the surface gravity defined with respect to the Killing vector $\partial_{\tau}$, which coincides with the standard surface gravity $\kappa$ of the horizon in the case that $v(\rho) \rightarrow 0$ as $\rho \rightarrow \infty$. For spacetimes in which $v(\rho)$ approaches a nonzero constant $v_{\infty}$, $\partial_{\tau}$ has norm $\left(1-v_{\infty}^{2}\right)^{1 / 2}$ at spatial infinity, so

$$
\kappa_{v}=\kappa\left(1-v_{\infty}^{2}\right)^{1 / 2} .
$$

For the example of a Schwarzschild black hole of mass $M$, the frame that falls from rest at infinity has $v(\rho)=-(2 G M / \rho)^{1 / 2}$. In this case the surface gravity is $\kappa=1 / 4 G M$, and the area radius $r(\rho)$ is equal to $\rho$, the radial proper distance measured at constant $\tau 5$

When a black hole forms from collapse of matter, the quantum state of any relativistic field tends towards the Unruh vacuum [24, 25]. This stationary state coincides with the vacuum as defined by freely falling $(\mathrm{FF})$

\footnotetext{
${ }^{4} \mathrm{~A}$ coordinate independent local expression for $\kappa_{v}$ is $(1 / 2)\left(s \cdot \nabla \xi^{2}\right) /(s \cdot \xi)^{2}$, where $s$ is the outward radial unit spacelike vector orthogonal to the free-fall worldline at the horizon and $\xi$ is the Killing vector with arbitrary normalization (which cancels out in this ratio).

${ }^{5}$ The vanishing of $r^{\prime \prime}(\rho)$ is equivalent to the vanishing of the (ingoing or outgoing) radial null-null component of the Ricci tensor. The condition $r^{\prime}(\rho)=1$ holds only if $v(\rho)$ vanishes at infinity.
} 
observers for modes with FF frequencies much larger than the surface gravity $\kappa$. In this state fluctuations on either side of the horizon are correlated in such a way that when tracing over the inside degrees of freedom, the reduced density matrix for the outgoing modes has the thermal form $\rho_{\text {ext }}=Z^{-1} \exp \left(-H / T_{H}\right)$, where $H$ is the Hamiltonian generating the Killing time translations (normalized at infinity) and $T_{H}=\kappa / 2 \pi$ is the Hawking temperature [26, 27, 28]. (Here and below we adopt units with $\hbar=c=1$.) Some of the outgoing thermal quanta reach spatial infinity and constitute Hawking radiation. Most of them however fall back across the horizon into the black hole, forming a thermal "atmosphere" 29]. The entropy of this atmosphere $S_{\text {atmo }}$ consists of the part of the entanglement (von Neumann) entropy $-\operatorname{Tr}\left(\rho_{\text {ext }} \ln \rho_{\text {ext }}\right)$ contributed by the near-horizon modes, that is, not including the entropy of the few low angular momentum Hawking quanta that escape to infinity. $S_{\text {atmo }}$ is in fact dominated by initially outgoing modes with large angular momenta which are turned back by the angular momentum barrier very close to the horizon.

Given the symmetries and the thermal character of the reduced density matrix $\rho_{\text {ext }}$, the atmosphere entropy can be expressed as a mode sum in the form

$$
S_{\text {atmo }}=\int d \omega \sum_{\ell=0}^{\infty}(2 \ell+1) n(\omega, \ell) s_{\text {therm }}\left(\omega / T_{H}\right),
$$

where $\omega$ is the Killing frequency, $\ell$ is the angular momentum, $s_{\text {therm }}\left(\omega / T_{H}\right)$ is the thermal entropy per mode 6 and $n(\omega, \ell)$ is the density of outgoing modes entangled to inside configurations.

The entropy diverges for two independent reasons. First, for each fixed $\omega$ and $\ell$, the mode density has a UV divergence logarithmic in the radial momentum cut-off. This arises from the infinite number of mode oscillations caused by the infinite blue shift at the horizon. Secondly, the sum over angular momenta is unbounded, producing a UV divergence quadratic in the angular momentum. Notice that for a $1+1$ dimensional black hole only the first, logarithmic divergence is present.

\footnotetext{
${ }^{6}$ Strictly speaking there is a weak $\ell$-dependence of the mode entropy due to the transmitted part of the infalling modes. These start from infinity in the ground state and partially penetrate the effective potential barrier, whereas the reflected parts of the nearhorizon outgoing modes are thermally populated. Since the transmitted part vanishes for $\ell \gg \omega / \kappa$, this $\ell$ dependence can be safely neglected when computing the entropy of the atmosphere.
} 


\section{Freely falling cut-off}

We are interested in regulating the atmosphere entropy by modifying the field propagation at high momenta as defined in a freely falling frame. This is in contrast to 't Hooft's "brick wall" approach [7] to regulating the entropy, where the cut-off is defined on static time slices. We first consider the simple case of a hard momentum cut-off.

Since the momentum cut-off is stationary and spherically symmetric, the atmosphere entropy still has the form (5). The entropy per mode is unchanged, but the mode density $n(\omega, \ell)$ is cut-off. To compute the atmosphere entropy we therefore only need to evaluate the cut-off mode density $n_{\text {cut }}(\omega, \ell)$. The calculation is identical for any free-fall frame in which the cutoff is imposed, so for notational simplicity in the following we adopt the one for which $v_{\infty}=0$.

We consider a massless scalar field in the spacetime with line element (22). The field theoretic justification for our evaluation of the entropy, while essential for a full understanding, is sequestered in the Appendix so as not to obscure the conceptual structure and simplicity of the entropy evaluation via WKB methods. The reader may wish to read the Appendix at this point before coming back to the main flow of the entropy computations.

The modes of fixed frequency and angular momentum take the form

$$
\varphi_{\omega \ell m}=\exp (-i \omega \tau) r^{-1} u_{\omega \ell}(\rho) Y_{\ell m}(\theta, \phi) .
$$

Since the relevant modes have very short wavelength, the radial mode functions $u_{\omega \ell}(\rho)$ are accurately described by the WKB form $u \sim \exp \left(i \int k d \rho\right)$, where the radial momentum $k=k_{\omega \ell}(\rho)$ obeys the relativistic dispersion relation $g^{\mu \nu} k_{\mu} k_{\nu}=0$, which here takes the form

$$
(\omega-v(\rho) k)^{2}=k^{2}+\ell(\ell+1) / r^{2} .
$$

The quantity $\Omega=\omega-v k$ is the free-fall frequency, whereas $\omega$ is the conserved Killing frequency. We introduce the UV cutoff by counting only modes with $k<\Lambda$ for some fixed $\Lambda \gg \kappa$. The cutoff is imposed on the radial component of momentum only, while the transverse (angular) momentum is unrestricted. It turns out however that the result would differ little if the cutoff were imposed on the total momentum, since the angular momentum barrier imposes an effective transverse momentum cut-off of order $(\omega \Lambda)^{1 / 2} \ll$ $\Lambda$.

The atmosphere entropy is defined as the entropy of the density matrix obtained after tracing over the field degrees of freedom localized inside the 
horizon. We shall evaluate the entropy here with the help of the WKB approximation, in which the modes are characterized by a well-defined momentum $k_{\omega \ell}(\rho)$, the solution of (7), at any radial position $\rho$. This allows us to count modes that lie outside the horizon and below the momentum cutoff.

The relevant mode density is the inverse of the frequency gap $\Delta \omega$ that separates two neighboring modes which are orthogonal with respect to the one-particle Hilbert space inner product restricted to the black hole exterior. As shown in the Appendix, in the WKB approximation, this mode density is given by

$$
n(\omega, \ell)=(2 \pi \kappa)^{-1} \ln \left(k_{\max } / k_{\min }\right),
$$

where $k_{\max }$ is the cutoff wavevector $\Lambda$ and $k_{\min }$ is the value of $k_{\omega \ell}(\rho)$ when the trajectory crosses the horizon after having reflected from the angular momentum barrier (see Fig. 1). In the near horizon region, the wavevector decreases exponentially as $\exp (-\kappa \tau)$ from $k_{\max }$ to $k_{\min }$ along the trajectory, thereby allowing to write (8) as $n(\omega, \ell)=\Delta \tau / 2 \pi$.

We now explain why it suffices to restrict attention to the near horizon region. At the turning points of the classical trajectories the dispersion relation (7) implies

$$
1-v_{\mathrm{t} . \mathrm{p} .}^{2} \simeq(\omega / p)^{2}
$$

where $p$ is the transverse momentum defined by

$$
p^{2}=\ell(\ell+1) / r^{2} .
$$

Since (as it turns out) the entropy is dominated by the high angular momentum modes with $p \gg \kappa$, and the contribution of high frequencies to the entropy is exponentially suppressed by $\exp (-2 \pi \omega / \kappa)$, we have $\left(1-v^{2}\right)_{\text {tp }} \ll 1$. It therefore suffices to use the linear approximation (3), and to set $r=r_{H}$ in the definition (10) of the transverse momentum $p$.

The dispersion relation (17) at the horizon yields $k_{\min }=\left(p^{2}-\omega^{2}\right) / 2 \omega$. The mode density (8) in the presence of the cut-off is therefore given by

$$
n_{\text {cut }}(\omega, \ell)=(2 \pi \kappa)^{-1} \ln \left(\frac{2 \omega \Lambda}{p^{2}-\omega^{2}}\right)
$$

The condition that $k_{\min }$ be less than $k_{\max }=\Lambda$ constrains $p$ to be less than

$$
p_{\max }=\sqrt{2 \omega \Lambda+\omega^{2}},
$$

thereby eliminating the quadratic divergence in (5) that comes from the angular momentum sum. For transverse momenta $p$ close to $\omega$ the contribution to the entropy is not well determined by our near horizon approximation 

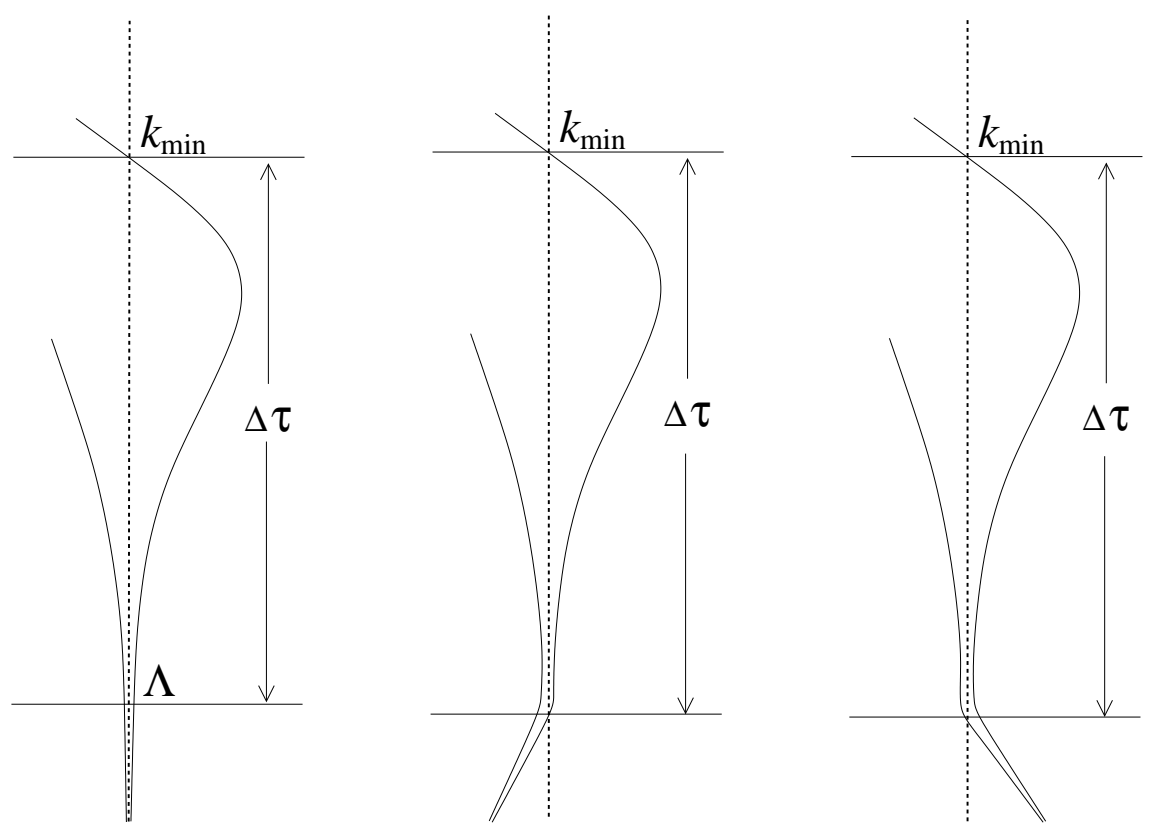

Figure 1: Trajectories of the particle and partner in the relativistic and dispersive cases. The density of states is always proportional to the PG time lapse $\Delta \tau$. In the relativistic case (left) this is the time the particle spends outside the horizon and below the hard cutoff. In the super-luminal case (center) it is simply the time the particle spends outside the horizon. In the sub-luminal case (right) it is the time between the horizon crossings of the partner and the particle. 
since, as shown by (9), the turning point is not near to the horizon. However, these momenta make a negligible contribution to the entropy. Thus we simply adopt the lower limit $p_{\text {min }}=\omega$ that is imposed by the condition $k_{\min }>0$, which is obeyed by any mode that is initially outgoing.

Since the atmosphere entropy is dominated by large angular momenta we can replace the sum over $\ell$ in (5) by an integral over $p^{2}$,

$$
\sum_{\ell}(2 \ell+1) \rightarrow r_{H}^{2} \int d p^{2}
$$

Then inserting (11) for the mode density into (5) yields

$$
S_{\text {atmo, cut }}=A \times(\kappa \Lambda) \times(2 \pi)^{-4} \int_{0}^{\infty} d x x s_{\text {therm }}(x),
$$

where $A$ is the horizon area $4 \pi r_{H}^{2}$ and $x=2 \pi \omega / \kappa$. (The integral is equal $3 \zeta(3) \simeq 3.6$. $)$ This expression gives the entropy of the black hole atmosphere when regulated by a hard momentum cut-off imposed in the freely falling frame that is at rest at infinity, evaluated using the WKB approximation. We now comment on several aspects of this result.

Frame dependence - Had we imposed the cut-off $\Lambda$ in a general free-fall frame the result would have been the same as (14), except with $\kappa$ replaced by $\kappa_{v}$ defined in (3) or equivalently (44). Thus the entropy actually depends on the free-fall frame in which the cut-off is defined. This is to be expected, since the cut-off prescription is not Lorentz invariant.

Universal dependence on transverse momentum cutoff-Although the entropy depends on the free-fall frame adopted for the cut-off $\Lambda$, it scales universally with the maximum angular momentum as

$$
\ell_{\max }^{2} \sim A \bar{p}_{\max }^{2}
$$

with $\bar{p}_{\max }=\sqrt{2 \kappa_{v} \Lambda}$ the value of $p_{\max }(12)$ evaluated at $\omega=\kappa_{v}$ (where most of the entropy resides). Thus each unit of area $\left(\bar{p}_{\max }\right)^{-2}$ carries of order one bit of entropy. Put differently, the entropy scales as the number of angular momentum states below the cutoff. The same scaling is found in the case of a dispersive cutoff in the next section.

Near-horizon approximation - As explained after (10), the near-horizon approximation is justified for modes with $p \gg \kappa$. We can now see that this is 
satisfied for the dominant modes, since $\bar{p}_{\max } \sim(\kappa \Lambda)^{1 / 2} \gg \kappa$.

Anisotropy of momentum cutoff-Since $\bar{p}_{\text {max }}$ is much smaller than the cut-off $\Lambda$, the entropy would be unchanged were the cut-off $\Lambda$ imposed isotropically on the norm of the momentum rather than on the radial component alone.

Mode density for dominant angular momenta - Since the entropy scales as the number of angular momentum states, the number of modes contributing to the entropy at fixed angular momentum $(\ell, m)$ is of order one for the dominating values of $\ell$. Indeed, evaluating the mode density (11) for $p$ parametrized as a fraction of the maximum value, $\bar{p}_{\max } / N$, yields $n_{\text {cut }}=$ $(\ln N) / 2 \pi \kappa$. Since thermal suppression by the Boltmann factor limits the effective frequency range to $\Delta \omega \sim \kappa / 2 \pi$, the number of contributing modes is $\sim n_{\text {cut }} \Delta \omega \sim(\ln N) /(2 \pi)^{2}$. Since most of the entropy comes from $N$ close to unity, only a "fraction of a mode" contributes for each angular momentum. In this regime $n_{\text {cut }} \Delta \omega$ should be interpreted as the probability to find the corresponding particle in the atmosphere outside the horizon.

Comparison with "brick wall" model - The entropy (14) scales as $\kappa \Lambda$, linearly with the momentum cutoff $\Lambda$. This scaling differs from that of the entropy regulated with a "brick wall" cutoff [7], i.e. a reflecting boundary condition at a proper distance $\epsilon$ from the horizon, which scales quadratically as $\epsilon^{-2}$. The difference arises only because different frames have been used to measure the cutoff scales: we measured $\Lambda$ on a time slice orthogonal to the free-fall worldlines, whereas $\epsilon$ was measured in Ref. [7] along a static time slice, orthogonal to the Killing vector. On the free-fall time slice, the same brick wall lies at a much smaller proper distance $\delta=\kappa \epsilon^{2} / 2$ from the horizon. Hence when expressed in terms of $\delta$, the brick wall entropy scales as $\delta^{-1}$, in agreement with what we found.

The agreement in overall scaling with the cutoff is a feature of the total entropy, after integrating over all frequencies and transverse momenta. This agreement arises because the dominant contributions scale the same way, despite the fact that the physical nature of the two cutoffs is different. In particular, the brick wall cuts off all modes at the same location, whereas we cut off all modes at the same momentum. In our case the cutoff location is not sharp, but can be characterized using the WKB approximation. Its value then depends on the frequency and angular momentum. The angular momentum dependence is negligible because, as noted after equation (12), $p \ll \Lambda$ for the frequencies $\omega \sim \kappa$ that dominate the total entropy. The frequency dependence on the other hand is significant. Using the dispersion 
relation (7) one finds near the horizon $k \simeq \omega / \kappa\left(\rho-\rho_{H}\right)$, so that the WKB position cutoff is $\omega / \kappa \Lambda$. However, this $\omega$ dependence does not affect the total entropy scaling since frequencies much greater than $\kappa$ are thermally suppressed. A further negligible difference with the brick wall evaluation arises because we cut off the WKB trajectories at the final horizon crossing, whereas the brick wall trajectories begin and end at the same radius, as explained in more detail in the Appendix.

\section{Dispersion induced cut-off}

We now remove the high wavevector cutoff $\Lambda$ and instead introduce dispersion into the field propagation by including higher space derivative terms suppressed by powers of $\Lambda$. Both super-luminal and sub-luminal dispersion will be considered. As explained above, it did not matter much whether the hard cut-off was isotropic or only in the radial direction. In the dispersive case the same will be true, at least in the super-luminal case. Whether it is true in the sub-luminal case will depend upon an open question to be discussed below.

The relativistic dispersion relation between frequency $\Omega$ and wavenumber $q$ for a massless field in flat spacetime is $\Omega^{2}=q^{2}$, where $q^{2}=\mathbf{q} \cdot \mathbf{q}$. We consider an isotropic UV-modified dispersion relation of the form

$$
\Omega^{2}=F^{2}(q)=\left(q \pm \frac{1}{n} q^{n} / \Lambda^{n-1}\right)^{2},
$$

chosen for convenience so that $F^{2}(q)$ is a perfect square. The quantity $\Lambda$ sets the scale for the nonlinear dispersion, and we assume $n>1$. At low wavenumbers $q \ll \Lambda$ the dispersion is relativistic. At high wavenumbers the group velocity $v_{g}=\partial_{q} \Omega$ is super-luminal for the plus sign and sub-luminal for the minus sign. At $q=\Lambda$ we have $\Omega(\Lambda)=\left(1 \pm \frac{1}{n}\right) \Lambda$ and $v_{g}(\Lambda)=1 \pm 1$. We now adopt units with $\Lambda=1$.

The dispersion relation has the given form only in a particular rest frame. As in the case of the hard cutoff we choose this to be the free-fall frame that is at rest at infinity. As in the nondispersive case, we can decompose the scalar field in the form (6) and use the WKB approximation for the radial functions $u_{\omega \ell}$. The positive free fall frequency outgoing $(k>0)$ mode with angular momentum $\ell$ satisfies the dispersion relation

$$
\begin{aligned}
\Omega=\omega-v(\rho) k & =\left(k^{2}+p^{2}\right)^{\frac{1}{2}} \pm \frac{1}{n}\left(k^{2}+p^{2}\right)^{\frac{n}{2}} \\
& \approx k+p^{2} / 2 k \pm k^{n} / n,
\end{aligned}
$$


where the approximate form in the last line holds when $p \ll k$ and $k \ll$ $\Lambda=1$. As can be verified a posteriori, this approximation is valid in the present context, as in the hard cutoff case. Moreover, the $r$ dependence in $p^{2}=\ell(\ell+1) / r^{2}$ can be safely replaced by the constant value $r_{H}$.

As before we take the field to be in the free fall vacuum state. A number of studies have demonstrated that in this state, to leading order in $\kappa / \Lambda$, models with UV dispersion reproduce the relativistic result that the outgoing modes at infinity are thermally populated at the Hawking temperature [30, [31, 32, 33]. To evaluate the entanglement entropy we need to know whether thermality extends to the near-horizon modes that contribute to the entropy. In the Appendix we deduce the quantum field theoretic description of the near-horizon free fall vacuum, generalizing to four dimensions the treatment of Ref. [34]. The state is a product of two-mode squeezed states that are thermal when reduced to one mode. We also discuss in the Appendix how the WKB approximation can be used to identify those entangled modes localized on either side of the horizon. In this section we shall assume that this WKB treatment provides an accurate evaluation of their density.

Under the above assumptions, the atmosphere entropy takes the same form (5) as before, but with a different density of modes $n_{\text {disp }}(\omega, \ell)$. As shown in the Appendix, the mode density is again given by (8). As in the relativistic case, the wavevector $k_{\text {min }}$ corresponds to the point where the particle trajectory finally crosses the horizon. In the WKB approximation, the only place where the dispersion enters the calculation of the atmosphere entropy is in the determination the initial wavevector $k_{\max }$.

In the super-luminal case the outgoing positive frequency modes emerge from behind the horizon after parting ways with their negative frequency partners which always remain behind the horizon (see Fig. 11). Therefore the relevant trajectory for determining the density of states to be included in the entropy extends between the two horizon crossing points of the positive frequency trajectory, and $k_{\max }$ is the value of $k$ at the first (outward) horizon crossing. The mode density at fixed $\omega$ and $\ell$ is thus finite, and the sum over $\ell$ is cut off when the turning point drops below the horizon and the two horizon crossing points merge. The atmosphere entropy is thus finite. We now discuss the determination of $k_{\max }$ in the sub-luminal case, before computing the entropy for both cases together.

In the sub-luminal case, the positive and negative frequency outgoing modes are initially infalling with respect to the static frame. For high enough frequency or low enough angular momentum the positive frequency modes convert to outward propagation at a turning point just outside the horizon (see Fig. 1), followed by a second turning point at the angular momentum 
barrier where they begin to fall back in. For lower frequency or higher angular momentum the pair of turning points disappears and the particle just follows the partner straight across the horizon with a small time lag. Since the entanglement of these modes is the source of the entropy, we shall identify $k_{\max }$ the value of $k$ for the positive frequency particle at the time the negative frequency partner crosses the horizon, leaving the particle stranded outside. This notion requires us to determine what is the particle momentum at a given PG time when the partner has a given momentum $k$. This determination is impossible if we refer only to Hamilton's equations, but in quantum mechanics the positive and negative frequency modes are linked through the specification of the in-modes which define the vacuum state. As shown in the appendix, by building wave packets of in-modes one can see that the particle and partner possess the same mean momentum at the same PG time. We therefore identify $k_{\max }$ as the value of the momentum when the partner crosses the horizon, see Fig. 1.

The mode density $n(\omega, \ell)$ of (8) is thus again finite, since $k_{\max } / k_{\min }$ is finite. There remains a potential divergence in the sum over angular momenta however since, unlike in the super-luminal case, this ratio remains strictly greater than unity. This is because the partner always crosses the horizon a finite time before the particle, as explained in the Appendix. The mode density therefore remains non-zero no matter how large $\ell$ becomes, and it may not approach zero fast enough for the sum over $\ell$ to converge. We will examine this potential divergence more closely later on.

The values of $k_{\max }$ and $k_{\min }$ are given by appropriate roots of the dispersion relation (18) evaluated at the horizon,

$$
\omega=p^{2} / 2 k \pm k^{n} / n
$$

The upper and lower signs are for the super- and sub-luminal cases respectively. This can be expressed in terms of the scaled variable $\tilde{k}=k /|\omega|^{\frac{1}{n}}$ as

$$
\sigma \tilde{k} \mp \frac{1}{n} \tilde{k}^{n+1}=p^{2} / 2|\omega|^{1+\frac{1}{n}},
$$

where $\sigma$ is the sign of $\omega$, positive for particle and negative for partner. The ratio $k_{\max } / k_{\min }$ is the same as the ratio of the $\tilde{k}$ 's, hence it depends upon $\omega$ and $p$ only through the combination $p^{2} / \omega^{1+\frac{1}{n}}$.

Following the above discussion, the mode density $n(\omega, \ell)$ in the superluminal case is determined by the $\log$ of the ratio of the two roots $\tilde{k}_{\text {max }}$ and $\tilde{k}_{\text {min }}$ of (20) for $\sigma=+1$ (particles) and the upper (negative) sign. The maximum transverse momentum is determined by the maximum of the left 
hand side of (20). It is given by

$$
p_{\max }=\sqrt{2 \omega \Lambda}\left(\frac{\omega}{\Lambda}\right)^{\frac{1}{2 n}}\left(\frac{n}{n+1}\right)^{\frac{n+1}{2 n}},
$$

where the explicit dependence on the momentum scale $\Lambda$ has been restored. This is essentially the same as in the case of the hard cutoff (12) in the limit $n \rightarrow \infty$, when the dispersion turns on suddenly at $k=\Lambda$. When $n$ is finite, $p_{\max }$ scales with a lower power of the "new physics" scale $\Lambda$ than for the hard cutoff. Therefore the atmosphere entropy will be similarly reduced.

In the sub-luminal case the lower sign applies, and $\tilde{k}_{\text {min }}$ is the root for $\sigma=+1$ (particle) while $\tilde{k}_{\max }$ is the root for $\sigma=-1$ (partner). In both cases, the mode density can be expressed as

$$
n_{\text {disp }}(\omega, \ell)=\frac{1}{2 \pi \kappa} \ln \left(\tilde{k}_{\max } / \tilde{k}_{\min }\right)=\frac{1}{2 \pi \kappa} \mathcal{N}_{n}^{ \pm}\left(\frac{p^{2}}{\omega \Lambda(\omega / \Lambda)^{\frac{1}{n}}}\right),
$$

where $\mathcal{N}_{n}^{ \pm}$is a function of its dimensionless argument that cannot be written in closed form. In Fig. 2 we plot this function for both sub-luminal $\left(\mathcal{N}_{n}^{-}\right)$ and super-luminal $\left(\mathcal{N}_{n}^{+}\right)$dispersion in the case $n=3$. Notice that while the cutoff scale is taken to be $\Lambda=10^{4} \kappa$, the maximal value of the transverse momentum in the super-luminal case is only $\simeq 25 \kappa$.

Using the mode density just obtained, the entropy (5) (again using (13)) evaluates to

$$
S_{\text {atmo }, \operatorname{disp}}=A \times \kappa \Lambda \times\left(\frac{\kappa}{\Lambda}\right)^{\frac{1}{n}} \times C_{n}^{ \pm},
$$

where the dependence on $\Lambda$ has been restored. The factor $C_{n}^{ \pm}$is a constant defined by

$$
C_{n}^{ \pm}=(2 \pi)^{-4-\frac{1}{n}}\left(\int_{0}^{y_{\max , n, \pm}} d y \mathcal{N}_{n}^{ \pm}(y)\right)\left(\int_{0}^{\infty} d x x^{1+\frac{1}{n}} s_{\text {therm }}(x)\right)
$$

where $y$ is the dimensionless variable $p^{2} /\left(2 \omega \Lambda(\omega / \Lambda)^{\frac{1}{n}}\right)$. The upper limit of integration $y_{\max , n,+}$ in the super-luminal case is the maximum value of the left hand side of (20), yielding a finite total entropy within the WKB treatment we have adopted. In the sub-luminal case $y_{\max , n,-}=\infty$, as explained above, hence the result depends on the asymptotic mode density at momenta larger than $\Lambda$.

The expression (23) gives the atmosphere entropy in the dispersive case. As in the case of the hard cutoff, the entropy scales universally with the square of the angular momentum cutoff, i.e. as $\ell_{\max }^{2} \sim A \bar{p}_{\max }^{2}$. In the 


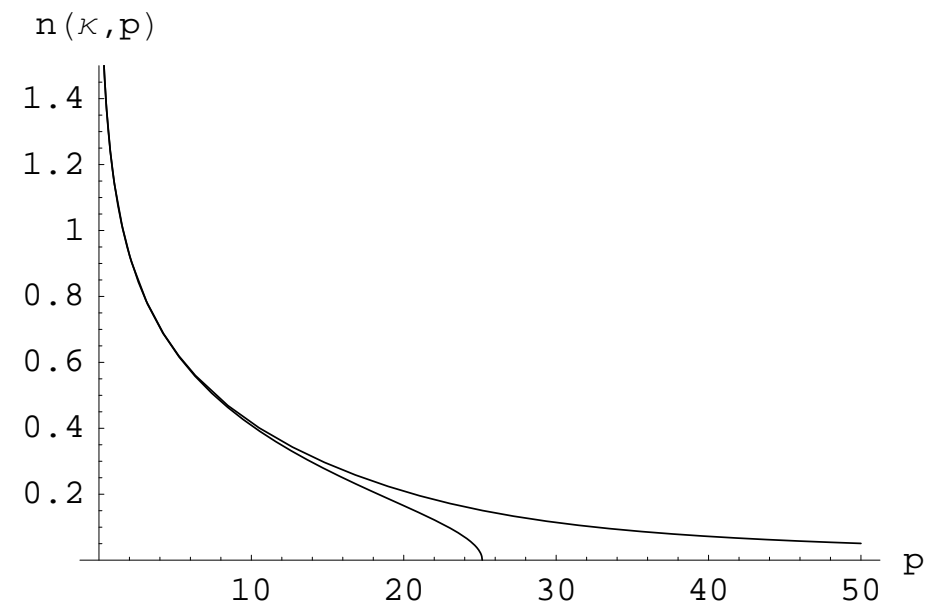

Figure 2: Plot of the mode density (22) vs. the transverse momentum $p$ at fixed frequency $\omega=\kappa$, for sub-luminal (upper curve) and super-luminal (lower curve) dispersion, with $\Lambda / \kappa=10^{4}$ and $n=3$, in units with $\kappa=1$.

present case, $\bar{p}_{\max }$ is the value of (21) at $\omega=\kappa$ (where most of the entropy resides). Comparing with our previous result (14) for the case of a hard cutoff, we note that the scale $\Lambda$ enters with a different power which depends on $n$. In the limit $n \rightarrow \infty$ one recovers the hard cutoff result, at least in the super-luminal case. This happens because for large $n$ the dispersion turns on abruptly at $k=\Lambda$.

Asymptotic mode density in sub-luminal case-The absence of an upper limit to the angular momentum sum in the sub-luminal case means that the result depends on the behavior of the dispersion relation at momenta higher than $\Lambda$. The dispersion relation (16) was introduced to parametrize only the first order correction to the relativistic dispersion. To analyze the mode density in the regime of very high momenta the first term in the Taylor expansion is inadequate, so we allow for a general dispersion relation of the form $(\omega-v k)^{2}=F^{2}$ where $F(k, p)$ is now an arbitrary function giving rise to sub-luminal propagation.

At fixed $k$ and $p$, as shown in the Appendix, the dispersion relation implies that the particle and partner locations near the horizon are separated by $\Delta \rho=2 \omega /(\kappa k)$, with the particle at larger $\rho$. The mode density is determined by the time between the horizon crossings of the partner and 
the particle, $\Delta \tau \approx|\Delta \rho /(d \rho / d \tau)|=2 \omega /\left(\kappa k\left(1-F_{, k}\right)\right)$. It is hence given approximately by

$$
n_{\mathrm{sub}}(\omega, \ell)=\frac{\omega}{\pi \kappa k\left(1-F_{, k}\right)},
$$

where $k$ satisfies the dispersion relation at the horizon, $\omega+k=F(k, p)$. As long as $F \gg \omega$, we have $k \simeq F(k, p)$ hence $n_{\text {sub }}$ behaves as $\sim \omega / F$ since for the sub-luminal case $F_{, k}$ is assumed less than one. The number of modes in the relevant thermal frequency range $\Delta \omega \sim \kappa$ is therefore $\sim$ $\kappa / F \ll 1$, assuming $F \gg \kappa$. That is, there is only a tiny probability to find a particle with large transverse momentum. The WKB approximation is probably unreliable in this regime, so our methods are unable to evaluate the contribution of these modes to the entropy.

\section{Discussion}

We have found that a freely falling hard momentum cutoff produces an entanglement entropy that scales as $A \kappa_{v} \Lambda$ with the cutoff $\Lambda$ and the surface gravity $\kappa_{v}$ defined in a given free fall frame. When the frame is taken to be at rest at infinity $\kappa_{v}$ coincides with the usual surface gravity $\kappa$. With no cutoff but instead sub- or super-luminal dispersion with index $n$ the scaling has an additional factor of $(\kappa / \Lambda)^{1 / n}$. If the freely falling cutoff $\Lambda$ is of the order of the Planck mass $M_{p}$ this entropy is negligible when compared to the Bekenstein-Hawking entropy $S_{\mathrm{BH}} \sim A M_{p}^{2}$ (for macroscopic black holes). This would conveniently eliminate the species problem, i.e. the nonuniversal contribution of the entanglement entropy to the black hole entropy, without the need to suppose a relation between the entanglement entropy and renormalization of the low energy effective Newton constant.

If instead the cutoff were given by $\Lambda=M_{p}^{2} / \kappa$ in the freely falling frame the scaling of the entanglement entropy would agree with $S_{\mathrm{BH}}$. This is equivalent to saying that the cutoff is actually $M_{p}$ in the static frame. While this might be considered natural from a "geometric" viewpoint, it seems unnatural from the viewpoint of the equivalence principle, which suggests that the cutoff should be a property of the freely falling vacuum. However, the equivalence principle would not forbid a cutoff arising from quantum gravity effects sensitive to the global properties of the background. In fact, there is some evidence for this.

Strong gravitational interactions between ingoing and outgoing quanta arise when the invariant mass squared reaches $M_{p}^{2}$ [7]. The radiative corrections associated with these interactions in the free fall vacuum outside the 
black hole were studied in Ref. [17, 18. Beyond the local effects that would also be present in the vacuum of flat spacetime, it was found that there are further contributions, dominated by in-modes with Killing frequency $\omega_{\text {in }} \sim \kappa$. These corrections induce a dissipating cutoff on the (backward) propagation of outgoing modes at the location where their frequency is $M_{p}^{2} / \kappa$ in the freely falling frame, i.e. $M_{p}$ in the static frame. While the significance of that preliminary analysis remains unclear, it does indicate a way in which the cutoff entanglement entropy might account for the Bekenstein-Hawking entropy.

It would be interesting to extend the considerations of the present paper to the cases of de Sitter and Rindler horizons. In the near-horizon limit these are identical to a black hole horizon, and a strong case can be made that they too posses a universal entropy density $1 / 4 L_{p}^{2}$ [35]. On the other hand, they differ in the nature of their "observer-dependence" and, perhaps more importantly, in the nature of the globally stationary free-fall frames that they admit. We leave the study of the regularized entanglement entropy for such horizons to future work.

We would like to reiterate that the WKB approach employed in this paper cannot provide conclusive results regarding the finiteness or scaling of the entanglement entropy in the dispersive cases, and it is particularly inconclusive in the sub-luminal case. The uncertainty arises since the mode density for the relevant modes is smaller than unity, so that we are not in the regime where a WKB treatment is guaranteed to be reliable. Therefore only a field theory analysis of the reduced density matrix outside the horizon would permit definitive conclusions to be reached.

In this paper we have studied the entanglement entropy between the degrees of freedom on either side of the sharp, low frequency horizon on a fixed black hole background. In the relativistic case, this horizon is a strict causal barrier, but in the dispersive cases it is dynamically distinguished only in the low frequency limit. (For sub-luminal dispersion the location of the barrier is "frequency dependent", while in the super-luminal case there is no barrier at all.) While this entanglement entropy is interesting, a more important, physical problem is to evaluate the "actual" thermodynamic or statistical entropy. The physically relevant definition of entanglement is not so clear in the dispersive cases, and in fact is unclear even in the relativistic case, once quantum fluctuations of the horizon are admitted. Presumably, the definition should ultimately be provided by a specified set of physical observers, interacting with the system in a definite manner.

Note added: While this paper was in press we became aware of a paper 
by Frolov and Novikov 37 in which a regulated black hole entanglement entropy for a massless scalar field was computed. A method similar to the one used here using WKB trajectories was employed to identify the modes contributing to the entropy. The cutoff was imposed by including only contributions from modes outside a certain minimum distance from the horizon, motivated by computations of quantum horizon fluctuations. The minimum distance was taken to be of the order the Planck length as measured on a static slice, so that the entropy scales with the area in Planck units.

\section{Acknowledgments}

This work was supported in part by the National Science Foundation under grants PHY-0300710 and PHY-0601800 through the University of Maryland, by the CNRS at the Institut d'Astrophysique de Paris, and by NATO grant CLG-979723. We are also grateful for the hospitality of both the University of Tours and Perimeter Institute, where some of this work was done.

\section{Appendix}

In this appendix we spell out the field theoretic rationale for our WKB evaluation of the mode density relevant to the entanglement entropy. The central ingredient is a description of the correlated structure of the near-horizon free-fall vacuum in terms of modes written in momentum space. These results could be useful in a future approach to computing the entanglement entropy without recourse to the WKB approximation.

\section{Action \& field equation}

We consider a massless scalar field in the spacetime with line element (2). It is governed by the action

$$
\begin{aligned}
I= & \frac{1}{2} \int d^{4} x \sqrt{-g} g^{\mu \nu} \partial_{\mu} \varphi \partial_{\nu} \varphi \\
= & \frac{1}{2} \int d \tau d \rho d \Omega\left\{r^{2}\left[\left(\left(\partial_{t}+v \partial_{\rho}\right) \varphi\right)^{2}-\left(\partial_{\rho} \varphi\right)^{2}\right]\right. \\
& \left.\quad-\left(\partial_{\theta} \varphi\right)^{2}-\frac{1}{\sin ^{2} \theta}\left(\partial_{\phi} \varphi\right)^{2}\right\}
\end{aligned}
$$


The field equation arising from this action is

$$
\left(\partial_{\tau}+\partial_{\rho} v\right) r^{2}\left(\partial_{\tau}+v \partial_{\rho}\right) \varphi-\partial_{\rho} r^{2} \partial_{\rho} \varphi-\frac{1}{\sin \theta} \partial_{\theta} \sin \theta \partial_{\theta} \varphi-\frac{1}{\sin ^{2} \theta} \partial_{\phi}^{2} \varphi=0
$$

\section{Modes}

Modes of fixed frequency and angular momentum take the form

$$
\varphi_{\omega \ell m}=\exp (-i \omega \tau) r^{-1} u_{\omega \ell}(\rho) Y_{\ell m}(\theta, \phi) .
$$

To find the equation satisfied by the mode function $u_{\omega \ell}$ we compute

$$
\partial_{\rho} r^{2} \partial_{\rho} r^{-1}=r \partial_{\rho}^{2}-r^{\prime \prime}
$$

and

$$
\left(-i \omega+\partial_{\rho} v\right) r^{2}\left(-i \omega+v \partial_{\rho}\right) r^{-1}=r\left(-i \omega+\partial_{\rho} v\right)\left(-i \omega+v \partial_{\rho}\right)-\left(v^{2} r^{\prime}\right)^{\prime}
$$

where the prime' denotes $\partial_{\rho}$. The mode equation is thus

$$
\left[\left(-i \omega+\partial_{\rho} v\right)\left(-i \omega+v \partial_{\rho}\right)-\partial_{\rho}^{2}+V_{\ell}(\rho)\right] u_{\omega \ell}=0
$$

with

$$
V_{\ell}(\rho)=\frac{\left(1-v^{2}\right) r^{\prime \prime}}{r}-\frac{2 v v^{\prime} r^{\prime}}{r}+\frac{\ell(\ell+1)}{r^{2}} .
$$

This is written for the general line element (2). With the Painlevé-Gullstrand (PG) coordinates for the Schwarzschild metric we would have $r(\rho)=\rho$, so the first term in $V_{\ell}$ vanishes and the second term becomes just $-2 v v^{\prime} / r$.

\section{Near-horizon approximation}

We are interested in modes propagating very near the horizon since these are the ones that contribute most of the entanglement entropy. Expanding about the horizon in $x=\rho-\rho_{H}$ we have

$$
\begin{aligned}
v(\rho) & =-1+\kappa_{v} x+\cdots \\
V_{\ell}(\rho) & =\frac{2 \kappa_{v} r^{\prime}(0)}{r_{H}}+\frac{\ell(\ell+1)}{r_{H}^{2}}+V^{\prime}(0) x+\cdots
\end{aligned}
$$

where $\kappa_{v}=v^{\prime}(0)$ is the surface gravity. The higher order terms in $x$ are negligible in the near-horizon region, and the $O(x)$ term is only significant when multiplied by a large coefficient. The derivatives $\partial_{\rho}$ in the first term 
of (A.6) provide large coefficients for the modes of interest, due to the large redshift at the horizon, so we retain the $O(x)$ contribution to the velocity (A.8) in that term. Since the entanglement entropy is dominated by large angular momenta, we can drop from $V_{\ell}$ all but the $\ell$-dependent term. In the near-horizon region we can set this term equal to its value at the horizon, $V_{\ell}=p^{2}$, where we have introduced the notation

$$
p^{2}=\ell(\ell+1) / r_{H}^{2}
$$

for the transverse momentum squared evaluated at the horizon.

\section{Momentum space}

The momentum space modes $\tilde{u}(k)$ are related to $u(\rho)$ by Fourier transform,

$$
u(\rho)=(2 \pi)^{-1 / 2} \int d k \tilde{u}(k) \exp (i k \rho) .
$$

In momentum space the mode equation (A.6) becomes

$$
(\omega-k \hat{v})(\omega-\hat{v} k) \tilde{u}_{\omega \ell}=\left(k^{2}+\hat{V}_{\ell}\right) \tilde{u}_{\omega \ell},
$$

where $\hat{v}=v(\hat{\rho})$ and $\hat{V}_{\ell}=V_{l}(\hat{\rho})$ with $\hat{\rho}=i \partial_{k}$. In the near-horizon approximation, and for large $\ell$ this mode equation takes the simpler form

$$
(\omega-k \hat{v})(\omega-\hat{v} k) \tilde{u}_{\omega \ell}=F_{\ell}^{2}(k) \tilde{u}_{\omega \ell},
$$

where now

$$
\hat{v}=-1+\kappa_{v} \hat{\rho}=-1+i \kappa_{v} \partial_{\rho}
$$

and

$$
F_{\ell}^{2}(k)=k^{2}+p^{2} .
$$

To simplify the notation we now drop the subscript $v$ on $\kappa$.

Thanks to the identities

$$
\begin{aligned}
(\omega-\hat{v} k) k^{-i \omega / \kappa-1} e^{-i k / \kappa} u & =k^{-i \omega / \kappa-1} e^{-i k / \kappa}\left(-i \kappa k \partial_{k} u\right) \\
(\omega-k \hat{v})(\omega-\hat{v} k) k^{-i \omega / \kappa-1} e^{-i k / \kappa} u & =k^{-i \omega / \kappa-1} e^{-i k / \kappa}\left(-\kappa^{2} k^{2} \partial_{k}^{2} u\right)
\end{aligned}
$$

the $\omega$-dependence of the solutions to (A.13) can be factored out, so the solutions take the form

$$
\tilde{u}_{\omega \ell}=k^{-i \omega / \kappa-1} e^{-i k / \kappa} \chi_{\ell}(k),
$$


where $\chi_{\ell}$ obeys

$$
\begin{aligned}
-\kappa^{2} k^{2} \partial_{k}^{2} \chi_{\ell} & =F_{\ell}^{2} \chi_{\ell}, \\
& =\left(k^{2}+p^{2}\right) \chi_{\ell} .
\end{aligned}
$$

For $k \gg p$ the solutions are $\exp ( \pm i k / \kappa)$, while for $k \ll p$ they are $k^{\alpha}$ with $\alpha=\frac{1}{2} \pm \sqrt{\frac{1}{4}-p^{2} / \kappa^{2}}$. Only the first case is relevant for the modes outside the horizon however, since $k_{\text {min }} \simeq p^{2} / \omega$ which is much greater than $p$ for the modes relevant to the entropy.

It so happens that (A.19) is closely related to Bessel's equation, and is exactly solvable in terms of Bessel functions:

$$
\chi_{\ell}(k)=\sqrt{k} \mathcal{C}_{\nu}(k), \quad \nu=\sqrt{\frac{1}{4}-p^{2} / \kappa^{2}}
$$

where $\mathcal{C}=J, Y, H^{(1,2)}$. As shown below these solutions can be classified according to whether $\chi_{\ell}$ has positive or negative Wronskian

$$
W=-i\left(\chi_{\ell}^{*} \partial_{k} \chi_{\ell}-\left(\partial_{k} \chi_{\ell}^{*}\right) \chi_{\ell}\right)
$$

\section{Dispersion}

Now we consider modifying the field equation by the addition of higher spatial derivative terms in the local rest frame defined by the unit timelike vector field $w=\partial_{\tau}+v(\rho) \partial_{\rho}$. For the metric (2) this frame is orthogonal to the surfaces of constant $\tau$.

The dispersion of interest becomes important at high wavevectors, where "high" is specified by a momentum scale $\Lambda \gg \kappa$. Since the modes of interest are only important near the horizon, it is sufficient to study dispersion by a modification of the near-horizon mode equation. To be able to handle arbitrary dispersion, it is convenient to work in momentum space [31, 34]. Allowing for distinct modifications of (A.13) in the radial and transverse directions yields the modified near-horizon mode equation

$$
(\omega-k \hat{v})(\omega-\hat{v} k) \tilde{u}_{\omega p}=F^{2}(k, p ; \Lambda) \tilde{u}_{\omega p},
$$

where $F(k, p ; \Lambda)$ is a function determining the dispersion, which is assumed to be relativistic if $k, p \ll \Lambda$.

As before, the $\omega$-dependence of the solutions to (A.22) can be totally factored out, so the solutions again take the form (A.18),

$$
\tilde{u}_{\omega p}=k^{-i \omega / \kappa-1} e^{-i k / \kappa} \chi_{p, \Lambda}(k),
$$


but with $\chi_{\ell}$ replaced by $\chi_{p, \Lambda}$. The latter satisfies (A.19) with the new $F^{2}$ function:

$$
-\kappa^{2} k^{2} \partial_{k}^{2} \chi_{p, \Lambda}=F^{2}(k ; p, \Lambda) \chi_{p, \Lambda}
$$

\section{Klein-Gordon inner product}

On a constant $\tau$ slice the KG inner product in both the relativistic case and in the presence of dispersion is given by

$$
\left\langle\varphi_{1}, \varphi_{2}\right\rangle=i \int d \Omega d \rho r^{2}\left(\varphi_{1}^{*}\left(\partial_{\tau}+v \partial_{\rho}\right) \varphi_{2}-\left(\left(\partial_{\tau}+v \partial_{\rho}\right) \varphi_{1}^{*}\right) \varphi_{2}\right)
$$

because the dispersion we consider only affects the spatial part of the action. It is diagonal on angular momentum modes, and for partial waves (6) with the same angular momentum it takes the form

$$
\left\langle\varphi_{\omega^{\prime}}, \varphi_{\omega}\right\rangle=\int d \rho\left(u_{\omega^{\prime}}^{*}\left(\omega+i v \partial_{\rho}\right) u_{\omega}+\left(\left(\omega^{\prime}-i v \partial_{\rho}\right) u_{\omega^{\prime}}\right)^{*} u_{\omega}\right) .
$$

In momentum space it becomes

$$
\left\langle\varphi_{\omega^{\prime}}, \varphi_{\omega}\right\rangle=\int d k\left(\tilde{u}_{\omega^{\prime}}^{*}(\omega-\hat{v} k) \tilde{u}_{\omega}+\left(\left(\omega^{\prime}-\hat{v} k\right) \tilde{u}_{\omega^{\prime}}\right)^{*} \tilde{u}_{\omega}\right) .
$$

For solutions factorized as in (A.18) or in (A.23), we make use of (A.16) to obtain

$$
\left\langle\varphi_{\omega^{\prime}}, \varphi_{\omega}\right\rangle=-i \kappa \int d k k^{i \Delta \omega / \kappa-1}\left(\chi^{*} \partial_{k} \chi-\left(\partial_{k} \chi^{*}\right) \chi\right)
$$

where $\Delta \omega=\omega^{\prime}-\omega$. In addition, for any solutions of (A.19) or its dispersive modification (A.24), the Wronskian of $\chi$ is a constant. Thus, irrespective of angular momentum, dispersion, or WKB approximation, the KG overlap of two modes in the near-horizon region is proportional to

$$
\int d k k^{i \Delta \omega / \kappa-1}=\int d(\ln k) e^{i(\ln k) \Delta \omega / \kappa} .
$$

\section{Mode density and classical trajectories}

To compute the entanglement entropy we need to know the density $n(\omega, \ell)$ of modes exterior to the horizon. This is just the reciprocal of the gap in Killing frequency $\omega$ that separates one exterior mode from the nearest orthogonal exterior mode with the same angular momentum. Orthogonality here is defined with respect to the one-particle Hilbert space inner product, which is defined by the Klein-Gordon inner product. The mode density is 
thus the inverse of the minimal $\Delta \omega$ for which the overlap (A.29) vanishes, hence

$$
n(\omega, \ell)=(2 \pi \kappa)^{-1} \ln \left(k_{\max } / k_{\min }\right) .
$$

We implement the restriction to exterior modes through the limits of integration $k_{\max }$ and $k_{\min }$, which are determined using the WKB approximation.

In both the relativistic and dispersive cases $k_{\min }$ is the value of the WKB momentum $k_{\omega \ell}\left(\rho_{H}\right)$ at the horizon crossing where the trajectory of the particle falls back into the black hole after having reflected from the angular momentum barrier (see Fig. 1). The value of $k_{\max }$ is just the hard cutoff $\Lambda$ in the relativistic case, and in the dispersive case it is determined by the passage of the particle (or its partner) across the horizon, as explained in the text.

The dispersion relation associated with the mode equation (A.22) is

$$
\omega-v(\rho) k=F(k, p ; \Lambda)
$$

where we took the positive root to describe outgoing modes. The classical trajectories satisfy Hamilton's equations,

$$
\begin{aligned}
d \rho / d \tau & =\partial \omega / \partial k \\
d k / d \tau & =-\partial \omega / \partial \rho,
\end{aligned}
$$

where the Hamiltonian is given by $\omega(\rho, k ; \ell)$, solution of (A.31). In the nearhorizon region, where $v(\rho) \approx-1+\kappa x$, A.33 implies the simple relation

$$
d k / d \tau=-\kappa k,
$$

both in the relativistic and dispersive cases. The wavevector $k$ therefore decreases exponentially as $\exp (-\kappa \tau)$ along the trajectory, both before and after reflection from the angular momentum barrier (which for large $\ell$ lies in the near-horizon region). Notice that the same exponential law with $k>0$ applies to the trajectories of the (outgoing but trapped) partners characterized by the opposite Killing frequency $-\omega$. The sign of $k$ should therefore be thought as that of the FF frequency.

Using (A.34), the mode density (A.30) can thus be reexpressed in the simple form

$$
n(\omega, \ell)=(2 \pi)^{-1} \Delta \tau(\omega, \ell)
$$

where $\Delta \tau(\omega, \ell)$ is the lapse of PG time evaluated along the relevant part of the classical trajectory. Two near-horizon modes are therefore orthogonal 
with respect to the KG product if their frequencies are separated by $\Delta \omega=$ $2 \pi / \Delta \tau$, irrespectively of the value of $p$ and the dispersion law $F 7$

It is informative to compare our result with the mode density for the static "brick wall" reflecting boundary condition used by 't Hooft in Ref. [7]. In that model, the quantum field is required to vanish at the wall, i.e. at a fixed radius outside the horizon. 't Hooft expressed the mode density as the derivative of the number of radial nodes with respect to frequency, $\pi^{-1} \int d r\left(\partial k_{r} / \partial \omega\right)$, where $\omega$ is the frequency with respect to the Schwarzschild time coordinate. Using the group velocity relation $d r / d t=$ $\partial \omega / \partial k_{r}$ this becomes simply $\pi^{-1} \int d t$. The integral is the Schwarzschild time lapse along the classical trajectory that rises from the wall and stops at a turning point.

The one-bounce trajectory that rises and then falls back to the wall is time-symmetric in terms of the Schwarzschild time coordinate, so we may extend the integral over $t$ to the full round trip integral and divide by two. This results in an expression that looks identical to (A.35), but it differs in that the time coordinate is the Schwarzschild time rather than the PG $\tau$ coordinate in (2). However, if we choose $v(\infty)=0, \kappa_{v}=\kappa$ then these time coordinates differ only by a function of radius, so their lapses at any fixed radius are equal. A small discrepancy remains because, unlike t'Hooft's brick wall, our regularization scheme leads to a slightly asymmetric domain of integration. The final point of the trajectory defining our mode density is at horizon crossing on the inward branch, while the initial point lies outside the horizon where the momentum is equal to the cutoff. The lapse of time associated with the extra bit is of order $1 / \Lambda$ however, and is thus negligible for our purposes. Our cutoff mode density and that of the brick wall prescription would thus agree if the cutoff momentum occurred at the same location as the brick wall. The comparison of the brick wall and momentum cutoffs is discussed further in the text.

\footnotetext{
${ }^{7}$ The linear growth in time of the relevant mode density in A.35 is familiar from the behavior of $\Sigma_{\text {modes }}=(\Delta t / 2 \pi) \int d \omega \rho(\omega)$, the number of modes involved in transition probabilities (the Golden Rule). Less familiar is the peculiar fact that here this appears expressed as (A.30), coming from an integral over momenta of the form $\int d k / k$. This feature of near horizon states was previously noticed in [36] in the context of both the acceleration horizon of an Unruh detector, and when counting the number of Hawking quanta in terms of the free fall frequency they had near the horizon. See the discussion around Eq. (3.44) in 25] for a brief account of this.
} 


\section{Free-fall vacuum: localization of particles and partners}

It follows from (A.28) that the modes

$$
\tilde{\phi}_{\omega \ell}^{+}(k)=\theta(k) k^{-i \omega / \kappa-1} e^{-i k / \kappa} \chi_{\ell}(k),
$$

have positive norm if $\chi_{\ell}$ has a positive Wronskian. These are complete and orthogonal when $\omega$ ranges over all values, both positive and negative. They thus generate the Hilbert space of one-particle outgoing states. The outgoing free-fall vacuum is defined by the condition that it is annihilated by the destruction operator $\hat{a}_{\omega \ell}^{i n}=\left\langle\tilde{\phi}_{\omega \ell}^{+}, \hat{\phi}\right\rangle$ corresponding to these modes because their FF frequency $\Omega=\omega-v k$ is positive, see (A.16).

The Fourier transforms of the modes (A.36) have support on both sides of the horizon, even for $\ell=0$. At the quantum level this gives rise to correlations between field fluctuations on either side of the horizon in the free-fall vacuum. These correlations are best characterized using a different mode basis consisting of positive Killing frequency "particles" and negative Killing frequency "partners", as we now explain.

The key mathematical ingredient is the decomposition into terms analytic in the upper and lower half complex $k$-planes:

$$
\theta(k) k^{-i \omega-1}=\frac{1}{e^{\pi \omega}-e^{-\pi \omega}}\left[e^{\pi \omega}(k-i \epsilon)^{-i \omega-1}-e^{-\pi \omega}(k+i \epsilon)^{-i \omega-1}\right]
$$

where $\epsilon \rightarrow 0^{+}$. Here we have chosen the branch cut of the logarithm to run along the negative $k$ axis. (To simplify the notation we now adopt units with $\kappa=1$.) The Fourier transform of the first term vanishes for $x<0$, since it is analytic in the lower half $k$-plane and bounded as $|k| \rightarrow \infty$. Similarly the Fourier transform of the second term vanishes for $x>0$. This is not the whole story however, since the mode (A.36) contains the additional factor $e^{-i k} \chi_{\ell}(k) 8$

Let us first consider the localization in the relativistic case with vanishing angular momentum. In this case $\chi_{\ell=0}(k) \propto e^{ \pm i k}$. The solution with positive Wronskian is $e^{i k}$, which cancels the factor $e^{-i k}$ in the mode. Thus the mode

\footnotetext{
${ }^{8}$ For zero angular momentum modes $\chi_{0}=\exp ( \pm i k / \kappa)$ the sign of the Wronskian is \pm , and (A.16) becomes $(\omega-\hat{v} k) \tilde{u}_{\omega 0}= \pm k \tilde{u}_{\omega 0}$. Those modes with positive free-fall frequency and positive Wronskian therefore have only positive wave-vectors, i.e. they are outgoing. In the presence of small (large) angular momentum the would-be purely outgoing modes are partly (totally) reflected by the angular momentum barrier and fall back as ingoing modes. We can still identify these as "outgoing" however, in the sense that they have no incoming part far from the horizon. These are the modes that are relevant to the entanglement entropy. Those with large $\ell$ constitute the dominant ones.
} 
splits into a sum of two functions strictly localized inside and outside the horizon. If we allow for angular momentum, then the solution $\chi_{\ell}(k)$ with positive Wronskian is no longer just $e^{i k}$, but is rather given by the Hankel function, $k^{1 / 2} H_{\nu}^{(1)}(k)$ in (A.20). This introduces branch cuts which lead to the Fourier transform of $(k-i \epsilon)^{-i \omega-1} e^{-i k} k^{1 / 2} H_{\nu}^{(1)}(k)$ having support inside as well as outside the horizon. The part inside corresponds to the reflected wave (of low momentum $k \sim \kappa$ ) that has bounced off the angular momentum barrier.

The addition of dispersion at a scale $\Lambda$ further complicates the localization properties by modifying this time the UV part $k \sim \Lambda \gg \kappa$ of the waves. As shown in [25, 34] the story is qualitatively similar, except for a mixing of outgoing and ingoing modes that corresponds to an extra pair creation process arising from the UV propagation of the modes. The amplitude of this is exponentially suppressed by a factor $\sim e^{-\pi \Lambda / \kappa}$ in the case of quartic dispersion $F^{2}=k^{2}+k^{4} / \Lambda^{2}$, and can therefore be safely neglected for black holes with surface gravity much less than $\Lambda$.

When (A.37) is inserted into the mode expression (A.36) the resulting two terms have KG norms with opposite signs. This is best seen with the help of the identity

$$
(k \pm i \epsilon)^{-i \omega-1}=\theta(k) k^{-i \omega-1}-\theta(-k) e^{ \pm \pi \omega}(-k)^{-i \omega-1} .
$$

The contribution from $\theta(k)$ has positive norm while that from $\theta(-k)$ has negative norm, opposite aside from the factor $e^{ \pm \pi \omega}$. Hence $(k-i \epsilon)^{-i \omega-1}$ yields a positive norm mode if $\omega>0$, while $(k+i \epsilon)^{-i \omega-1}$ yields a negative norm mode. Taking into account the normalization factor of each of these modes, the annihilation operator for (A.36) with $\omega>0$ thus decomposes as

$$
\frac{1}{\sqrt{1-e^{-2 \pi \omega}}}\left[\hat{b}_{\omega \ell}-e^{-\pi \omega} \hat{c}_{\omega \ell}^{\dagger}\right] \text {. }
$$

That is, it is a combination of the annihilator of the $(k-i \epsilon)^{-i \omega-1}$ mode and the creation operator for the complex conjugate of the $(k+i \epsilon)^{-i \omega-1}$ mode, which is a positive norm mode with Killing frequency $-\omega$. When $\omega<0$, one finds (A.39) with $b$ and $c$ interchanged, and $\omega$ replaced by $-\omega$. The condition that the annihilator for (A.36) (for all $\omega$ ) annihilates the state thus becomes a statement that the state contains correlated pairs of $b, c$ particles with opposite Killing frequency. Tracing over the negative Killing frequency particles yields a thermal state at the Hawking temperature $T_{H}=\kappa / 2 \pi$. Thus, even in the dispersive case, the near horizon free fall vacuum has a thermal character. 
With vanishing angular momentum and no dispersion, these correlated pairs are strictly localized, with the positive Killing frequency one outside and the negative Killing frequency one inside the horizon. With angular momentum added, the positive Killing frequency mode falls back across the horizon with a low radial momentum. With dispersion, there is a further blurring of the outside/inside distinction which this time comes from the UV domain. Nevertheless, because $\Lambda \gg \kappa$ there exists an intermediate domain $\Lambda \gg k \gg \kappa$ wherein (A.37) can be used to define a decomposition into a correlated pair of positive and negative Killing frequency modes which are localized on either side of the horizon. Moreover, when wavepackets are formed by integrating over $\omega$, those wavepackets will spend some time near the horizon behaving as do the ones with no angular momentum and no dispersion. The amount of time the one with positive $\omega$ will spend outside the horizon while its partner is inside is (by definition) given by (A.35).

Indeed, in identifying the modes contributing to entanglement entropy, we use the WKB description of these modes, and require that the positive Killing frequency particle lies outside the horizon while the negative Killing frequency partner lies inside. Having formed a wavepacket, at any given time the wavevector of the particle and partner is given by the stationary point of the wavepacket integral over $\omega$. It follows from (A.37) and the fact that no other rapidly oscillating function of $\omega$ appears in the wavepacket that, at leading WKB order, this wavevector is the same for the particle and partner. That common wavevector is then used to locate the particle and partner using the dispersion relation with $\omega>0$ for the particle and $-\omega$ for the partner.

\section{Particle and partner trajectories}

In the near horizon region, the dispersion relation (A.31) reads

$$
\omega=\kappa k x-k+F(k, p ; \Lambda) .
$$

Solving for the trajectory $x=x(k, \omega, p ; \Lambda)$ we have

$$
x(\omega, p, k ; \Lambda)=x_{0}(k, p ; \Lambda)+\omega / \kappa k,
$$

with

$$
x_{0}(k, p ; \Lambda)=(k-F(k, p ; \Lambda)) / \kappa k .
$$

As just explained, the particle and partner have the same value of $k$ at any given time, and opposite values of $\omega$. Hence the mean position (A.42) of the 
pair is independent of $\omega$, while the separation of the particle and partner is universally given by

$$
\Delta x=2 \omega / \kappa k,
$$

independent of $p$ and the form of the dispersion. This reflects at the classical level the fact, seen in (A.23), that the modes always split into a factor independent of $p$ and $F$ and a factor independent of $\omega$. This result, as many others, is identical to what was found in the $1+1$ case studied in [34] because the transverse momentum $p$ only enters as a modification of the dispersion function $F$.

The space time trajectories $x(\tau)$ are determined by (A.41) since $k(\tau)$ is known. As seen in (A.34), the $k$-trajectories are simply given by $k(\tau)=$ $k_{0} \exp (-\kappa \tau)$, again independently of the value of $p$ and the dispersion relation governed by $F$.

\section{References}

[1] J. D. Bekenstein, "Black holes and the second law," Lett. Nuovo Cim. 4, 737 (1972).

[2] J. D. Bekenstein, "Black holes and entropy," Phys. Rev. D 7, 2333 (1973).

[3] S. W. Hawking, "Black holes and thermodynamics," Phys. Rev. D 13, 191 (1976).

[4] G. W. Gibbons and S. W. Hawking, "Action integrals and partition functions in quantum gravity," Phys. Rev. D 15, 2752 (1977).

[5] R. D. Sorkin, "On the entropy of the vacuum outside a horizon," in B. Bertotti, F. de Felice, and A. Pascolini (eds.), Tenth International Conference on General Relativity and Gravitation, Contributed Papaers, Vol. 2 (Consiglio Nazionale Delle Ricerche, 1983), 734.

[6] L. Bombelli, R. K. Koul, J. H. Lee and R. D. Sorkin, "A Quantum source of entropy for black holes," Phys. Rev. D 34, 373 (1986).

[7] G. 't Hooft, "On the quantum structure of a black hole," Nucl. Phys. B 256, 727 (1985).

[8] W. H. Zurek and K. S. Thorne, "Statistical mechanical origin of the entropy of a rotating, charged black hole," Phys. Rev. Lett. 54 (1985) 2171. 
[9] L. Susskind and J. Uglum, "Black hole entropy in canonical quantum gravity and superstring theory," Phys. Rev. D 50, 2700 (1994) arXiv:hep-th/9401070].

[10] T. Jacobson, "Black hole entropy and induced gravity," arXiv:gr-qc/9404039.

[11] J. G. Demers, R. Lafrance and R. C. Myers, "Black hole entropy without brick walls," Phys. Rev. D 52, 2245 (1995) [arXiv:gr-qc/9503003].

[12] D. Kabat, "Black hole entropy and entropy of entanglement," Nucl. Phys. B 453, 281 (1995) arXiv:hep-th/9503016.

[13] S. P. Kim, S. K. Kim, K. S. Soh and J. H. Yee, "Renormalized thermodynamic entropy of black holes," Phys. Rev. D 55, 2159 (1997) arXiv:gr-qc/9608015.

[14] S. N. Solodukhin, "One loop renormalization of black hole entropy due to nonminimally coupled matter," Phys. Rev. D 52, 7046 (1995) arXiv:hep-th/9504022.

[15] F. Larsen and F. Wilczek, "Renormalization of black hole entropy and of the gravitational coupling constant," Nucl. Phys. B 458, 249 (1996) arXiv:hep-th/9506066.

[16] T. Jacobson, "Thermodynamics of space-time: The Einstein equation of state," Phys. Rev. Lett. 75, 1260 (1995) [arXiv:gr-qc/9504004].

[17] R. Parentani, "Quantum metric fluctuations and Hawking radiation," Phys. Rev. D 63, 041503 (2001) arXiv:gr-qc/0009011.

[18] R. Parentani, "Beyond the semiclassical description of black hole evaporation," Int. J. Theor. Phys. 41 (2002) 2175; and Proceedings of the meeting "Analog models of General Relativity" published in Artificial Black Holes, Eds. M. Novello, M. Visser and G. Volovik, (World Scientific, 2002).

[19] R. D. Sorkin, "How wrinkled is the surface of a black hole?," arXiv:gr-qc/9701056.

[20] A. Casher, F. Englert, N. Itzhaki, S. Massar and R. Parentani, "Black hole horizon fluctuations," Nucl. Phys. B 484, 419 (1997) arXiv:hep-th/9606106. 
[21] D. Marolf, "On the quantum width of a black hole horizon," arXiv:hep-th/0312059.

[22] D. Chang, C. S. Chu and F. L. Lin, "Transplanckian entanglement entropy," Phys. Lett. B 583, 192 (2004) arXiv:hep-th/0306055.

[23] K. Martel and E. Poisson, "Regular coordinate systems for Schwarzschild and other spherical spacetimes," Am. J. Phys. 69, 476 (2001) arXiv:gr-qc/0001069.

[24] W. G. Unruh, "Origin of the particles in black hole evaporation," Phys. Rev. D 15, 365 (1977).

[25] R. Brout, S. Massar, R. Parentani and P. Spindel, "A Primer for black hole quantum physics," Phys. Rept. 260, 329 (1995).

[26] R. M. Wald, "On Particle creation by black holes," Commun. Math. Phys. 45, 9 (1975).

[27] L. Parker, "Probability distribution of particles created by a black hole," Phys. Rev. D 12, 1519 (1975).

[28] S. W. Hawking, "Breakdown of predictability in gravitational collapse," Phys. Rev. D 14, 2460 (1976).

[29] K. S. Thorne, W. H. Zurek, and R. H. Price, "The Thermal atmosphere of a black hole," Chapter 8 in Black Holes: The Membrane Paradigm, R. Price, K. Thorne, D.A. MacDonald, eds. (Yale University Press, 1986).

[30] W. G. Unruh, "Sonic analog of black holes and the effects of high frequencies on black hole evaporation," Phys. Rev. D 51, 2827 (1995).

[31] R. Brout, S. Massar, R. Parentani and P. Spindel, "Hawking radiation without transplanckian frequencies," Phys. Rev. D 52, 4559 (1995) arXiv:hep-th/9506121].

[32] S. Corley and T. Jacobson, "Hawking spectrum and high frequency dispersion," Phys. Rev. D 54, 1568 (1996) arXiv:hep-th/9601073.

[33] T. Jacobson, "Trans-Planckian redshifts and the substance of the space-time river," Prog. Theor. Phys. Suppl. 136, 1 (1999) arXiv:hep-th/0001085. 
[34] R. Balbinot, A. Fabbri, S. Fagnocchi and R. Parentani, "Hawking radiation from acoustic black holes, short distance and backreaction effects," Riv. Nuovo Cim. 28, 1 (2005) arXiv:gr-qc/0601079.

[35] T. Jacobson and R. Parentani, "Horizon Entropy," Found. Phys. 33, 323 (2003) arXiv:gr-qc/0302099.

[36] R. Parentani and R. Brout, "Physical interpretation of black hole evaporation as a vacuum instability," Int. J. Mod. Phys. D 1, 169 (1992).

[37] V. P. Frolov and I. Novikov, "Dynamical origin of the entropy of a black hole," Phys. Rev. D 48, 4545 (1993) arXiv:gr-qc/9309001. 